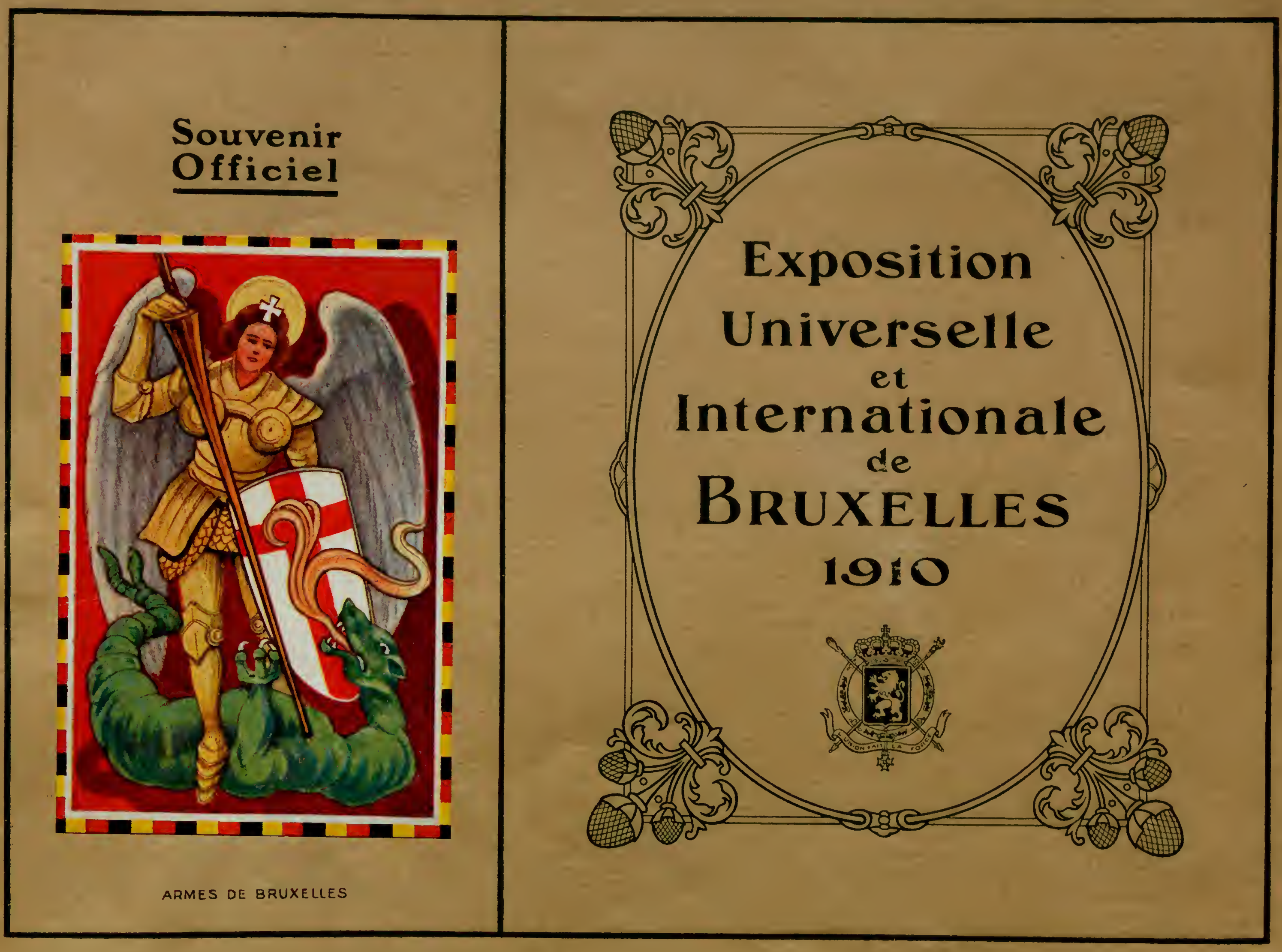





\section{ALBUM OFFICIEL}

DE LEXXPOSITION UNIVERSELLE ET INTERNATIONALE

\section{DE BRUXELLES 1910}

25 Vues Photographiques (Série VALEnTINE, Marque J. V.) 


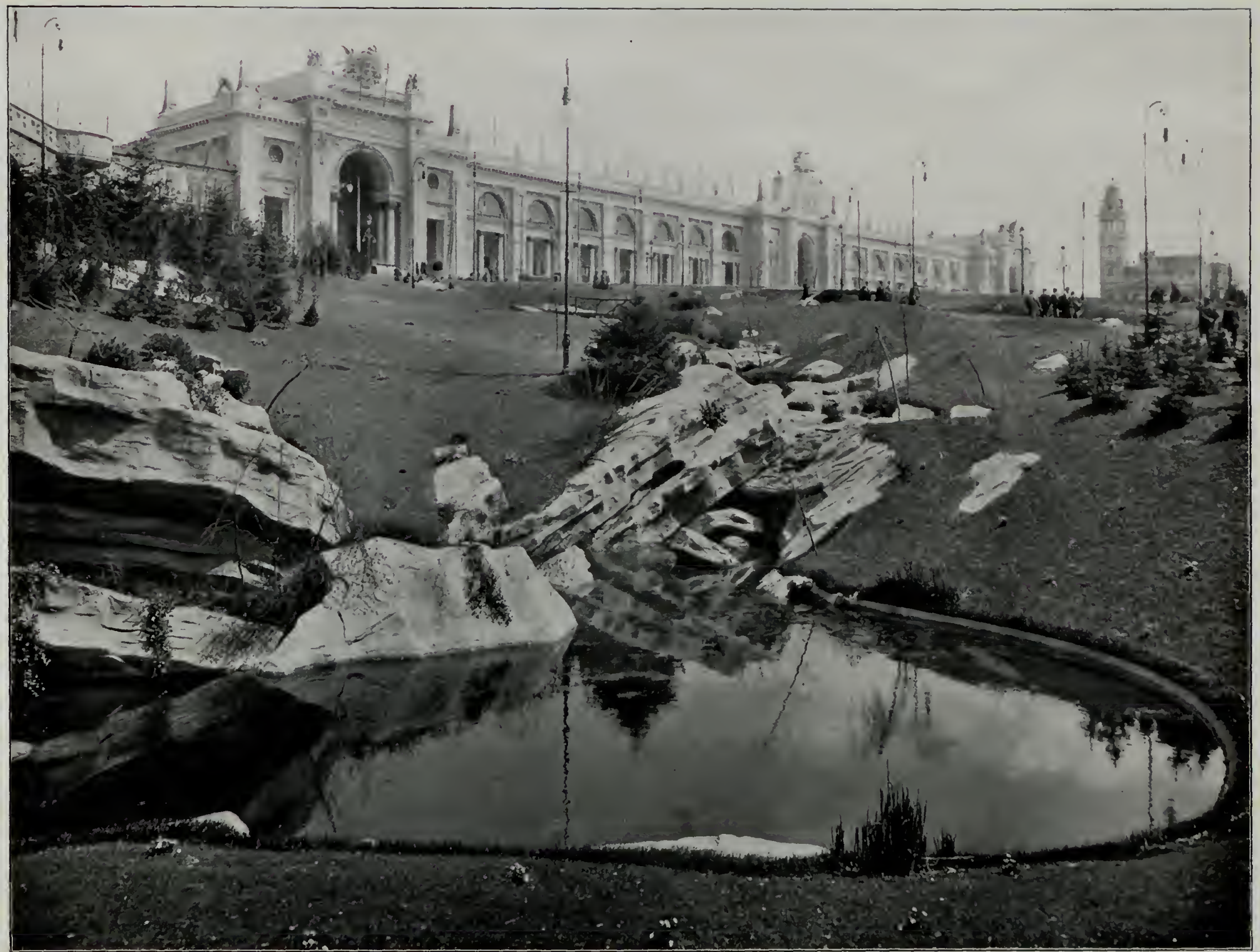

JARDIN SUISSE

La première vue que le visiteur rencontre en entrant par l'entrée principale. 


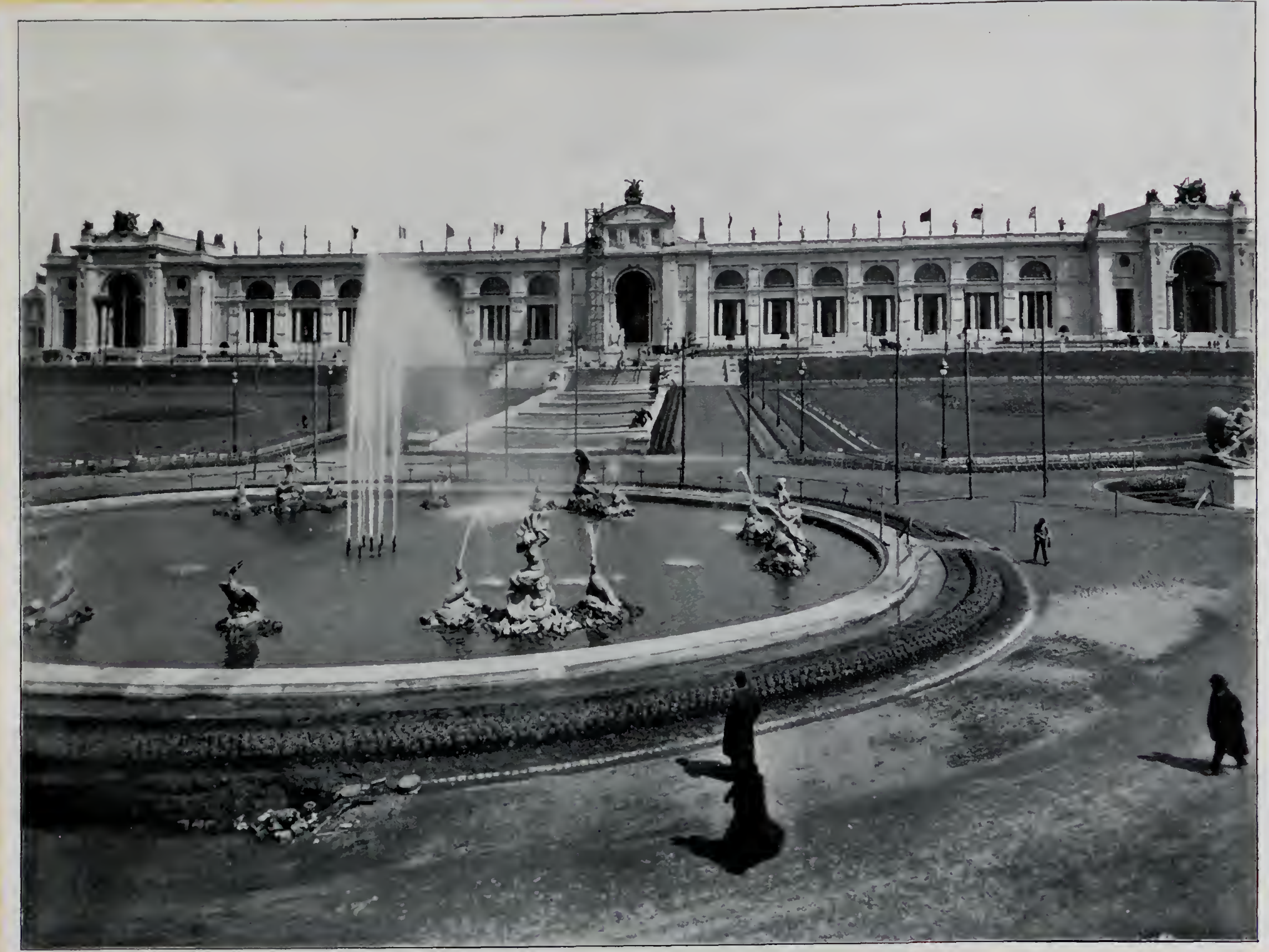

FAÇADE PRINCIPALE ET LA FONTAINE

La façade principale a été conçue en style classique, elle a un développement de plus de 250 mètres de longueur et une superficie de 6,500 mètres carrés. 

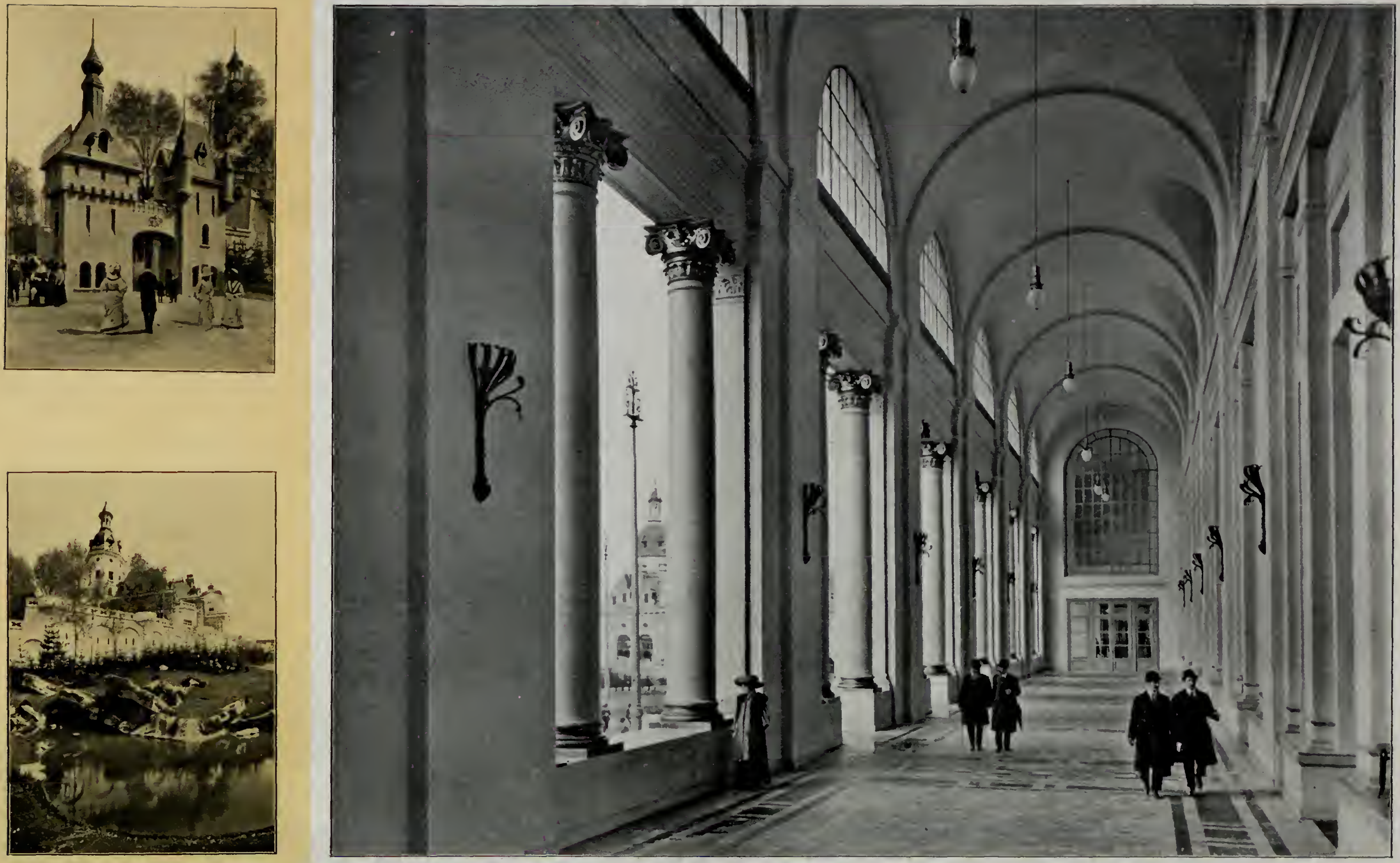

COLONNADE DE LA FAÇADE PRINCIPALE

De là on aperçoit une magnifique vue générale, ayant comme rideau le superbe Bois de la Cambre. La petite vue au-dessus représente la grande porte de Bruxelles Kermesse et la petite vue en-dessous le restaurant "Chien Vert». 

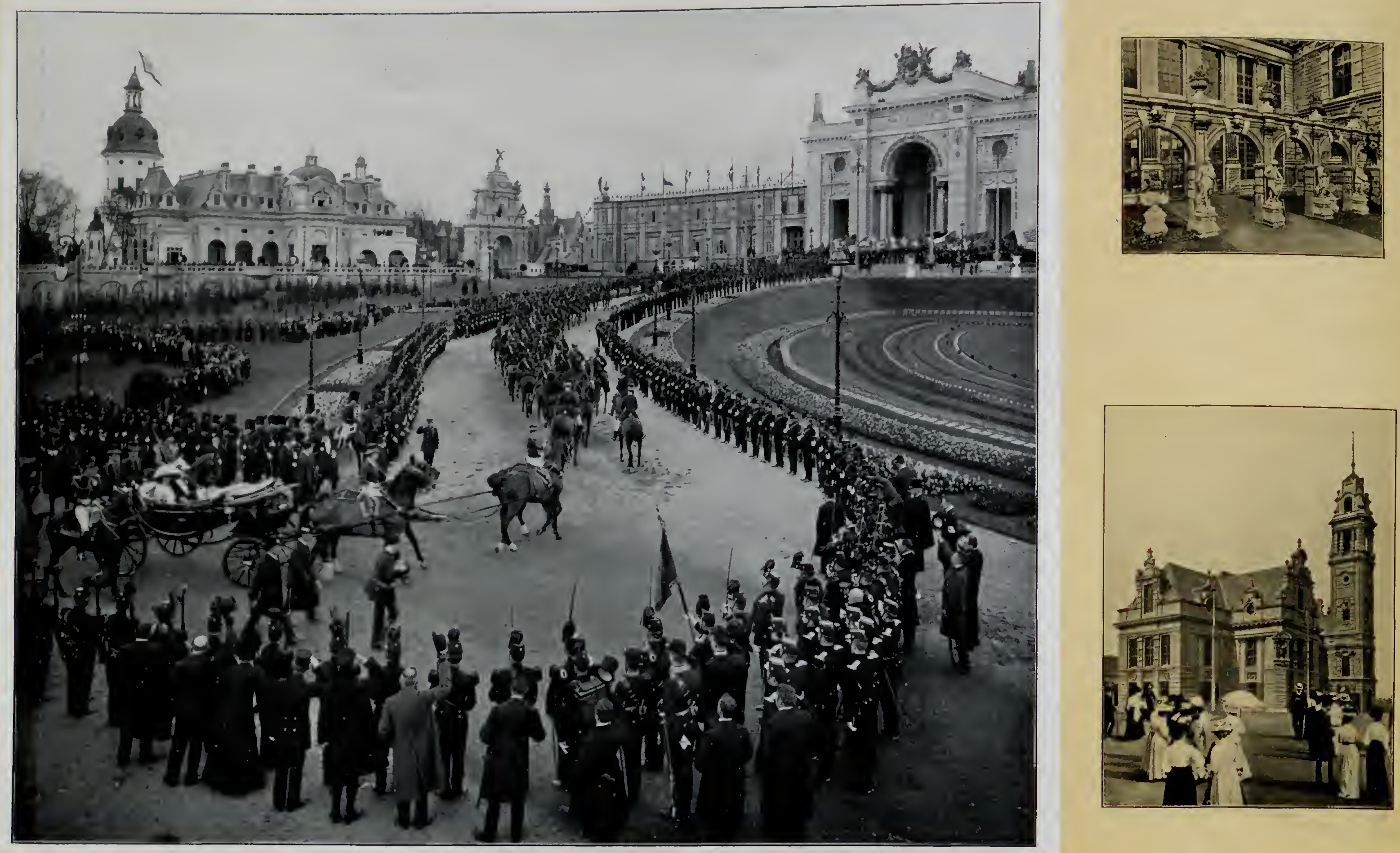

VISITE DU ROI ET DE LA REINE DES BELGES

Cette photographie a été prise à l'ouverture de l'Exposition. On peut remarquer dans le carrosse LL. MM. le Roi et la Reine des Belges. La petite vue au-dessus est une partie du beau Palais de la ville de Bruxelles, dont la petite vue en-dessous représente la vue générale. 

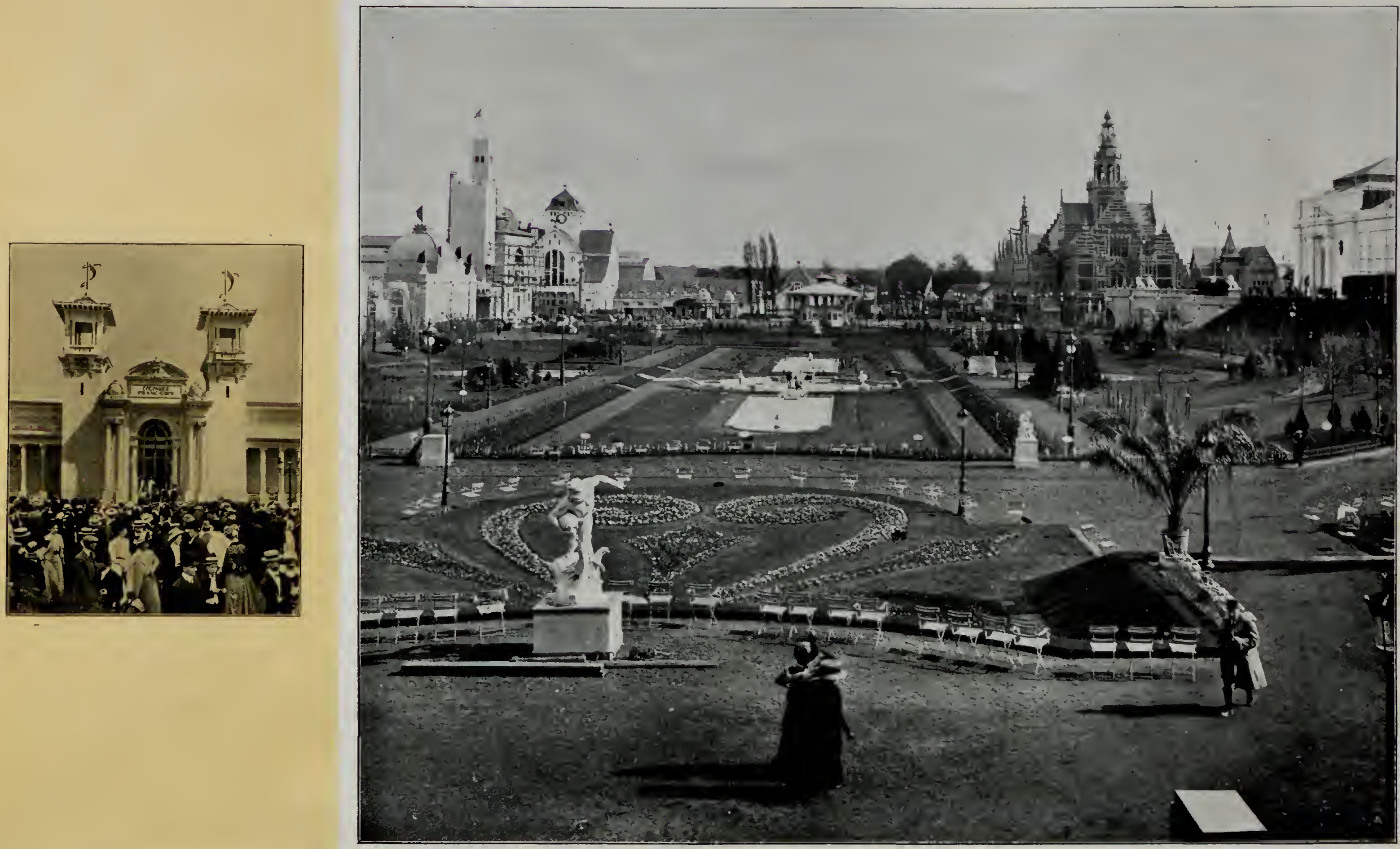

JARDINS DE LA VILLE DE PARIS

Cette vue s'aperçoit depuis les Halls des Nations. Dans le fond on remarque les tours des Pavillons d'Allemagne, Monaco et des Pays-Bas. La petite vue représente l'entrée du Pavillon des Colonies françaises. 


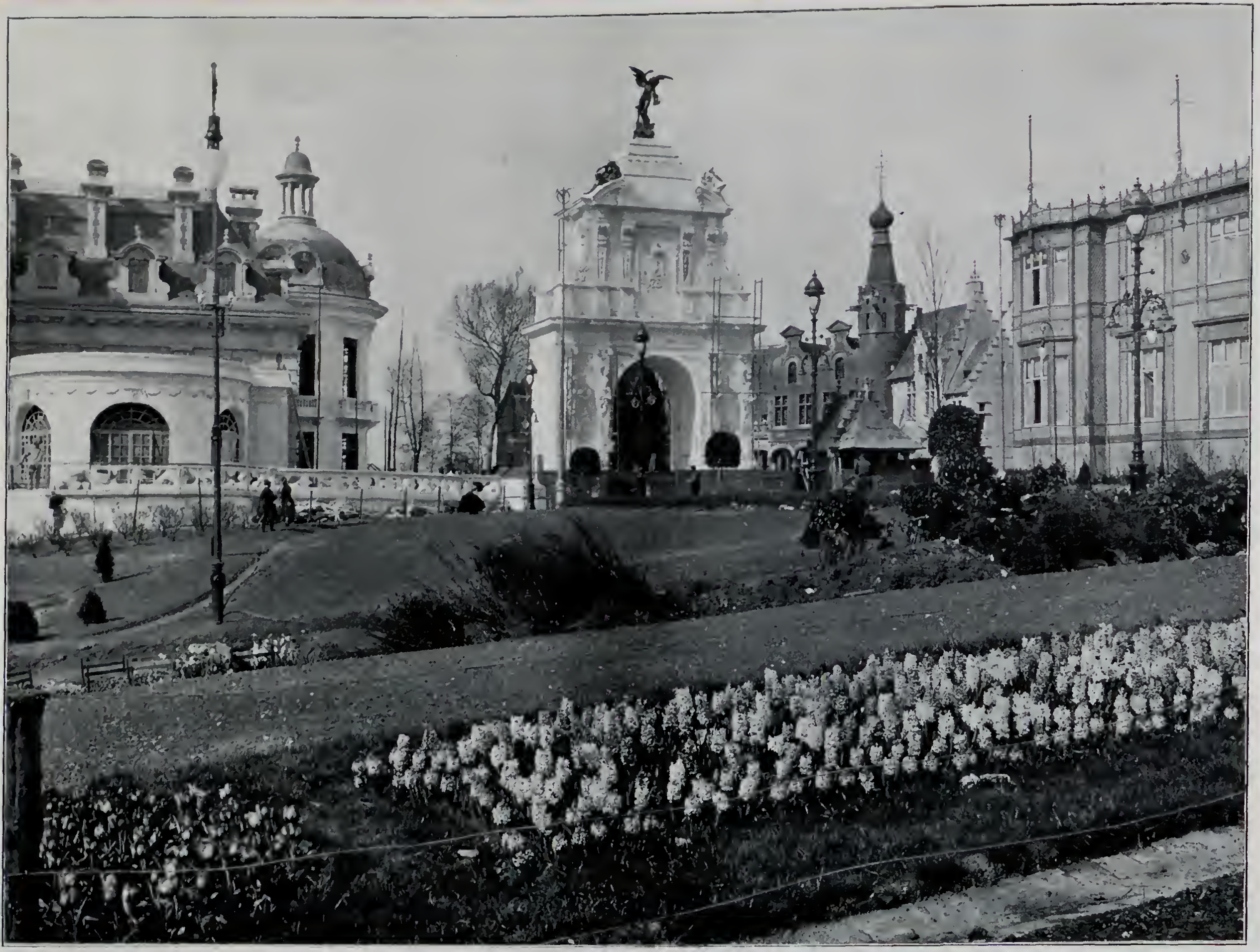

ENTRÉE PRINCIPALE DE BRUXELLES KERMESSE

Au milieu se trouve l'Arc de Triomphe; on aperçoit au-dessus la statue de Saint-Michel. Au premier plan on remarque un parquet de jacinthes faisant partie du Jardin de Bruxelles. 


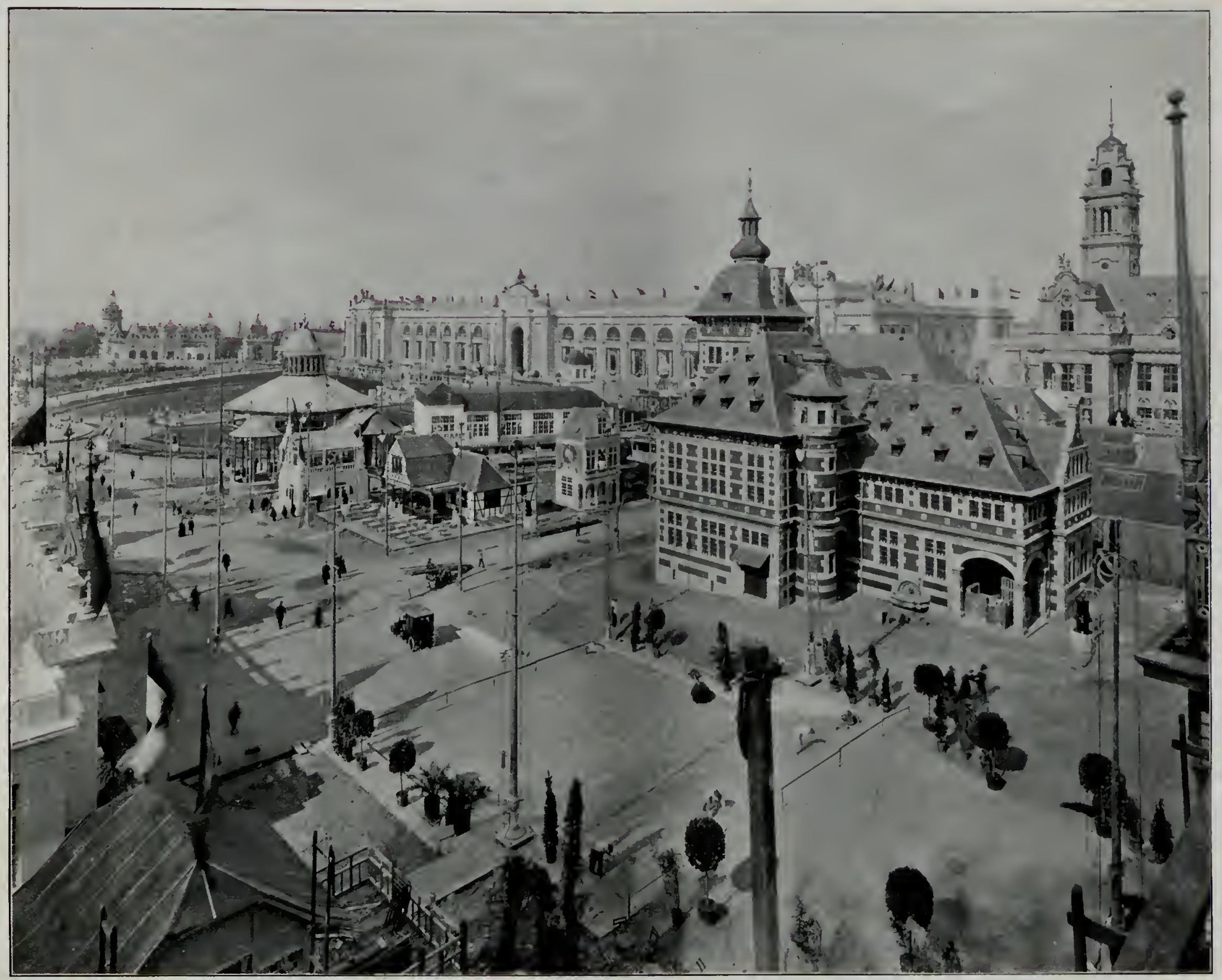

VUE GÉNÉRALE

Prise de la Salle des Fêtes. Le bâtiment à droite est le Pavillon de Liége, et dans le fond on voit le Grand Palais et une partie du Pavillon de Bruxelles. 


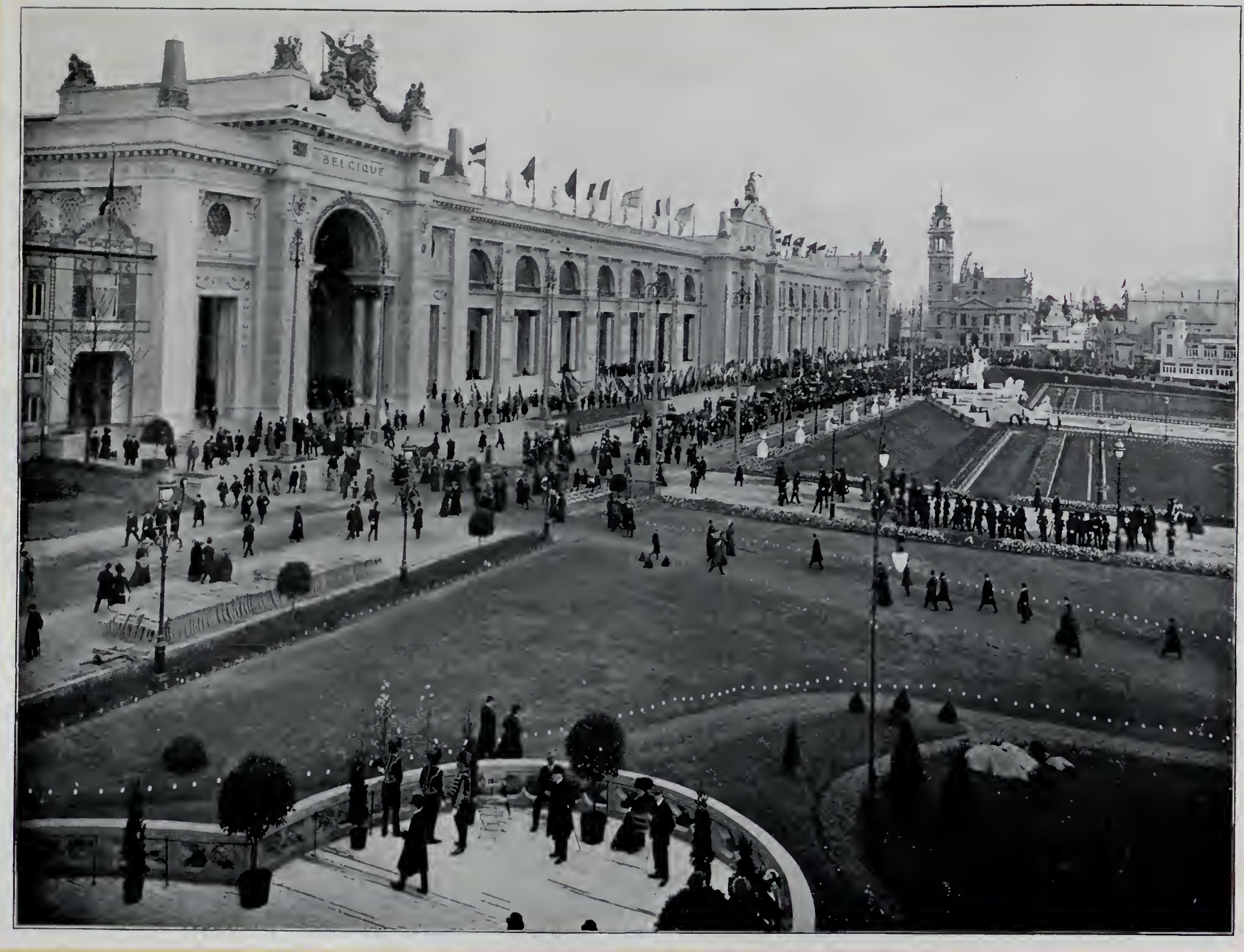

\section{LA GRANDE TERRASSE}

La grande entrée à gauche conduit directement à la Section britannique, en passant par la Section belge. Le bâtiment dans le fond est le Pavillon de la Ville de Bruxelles. 

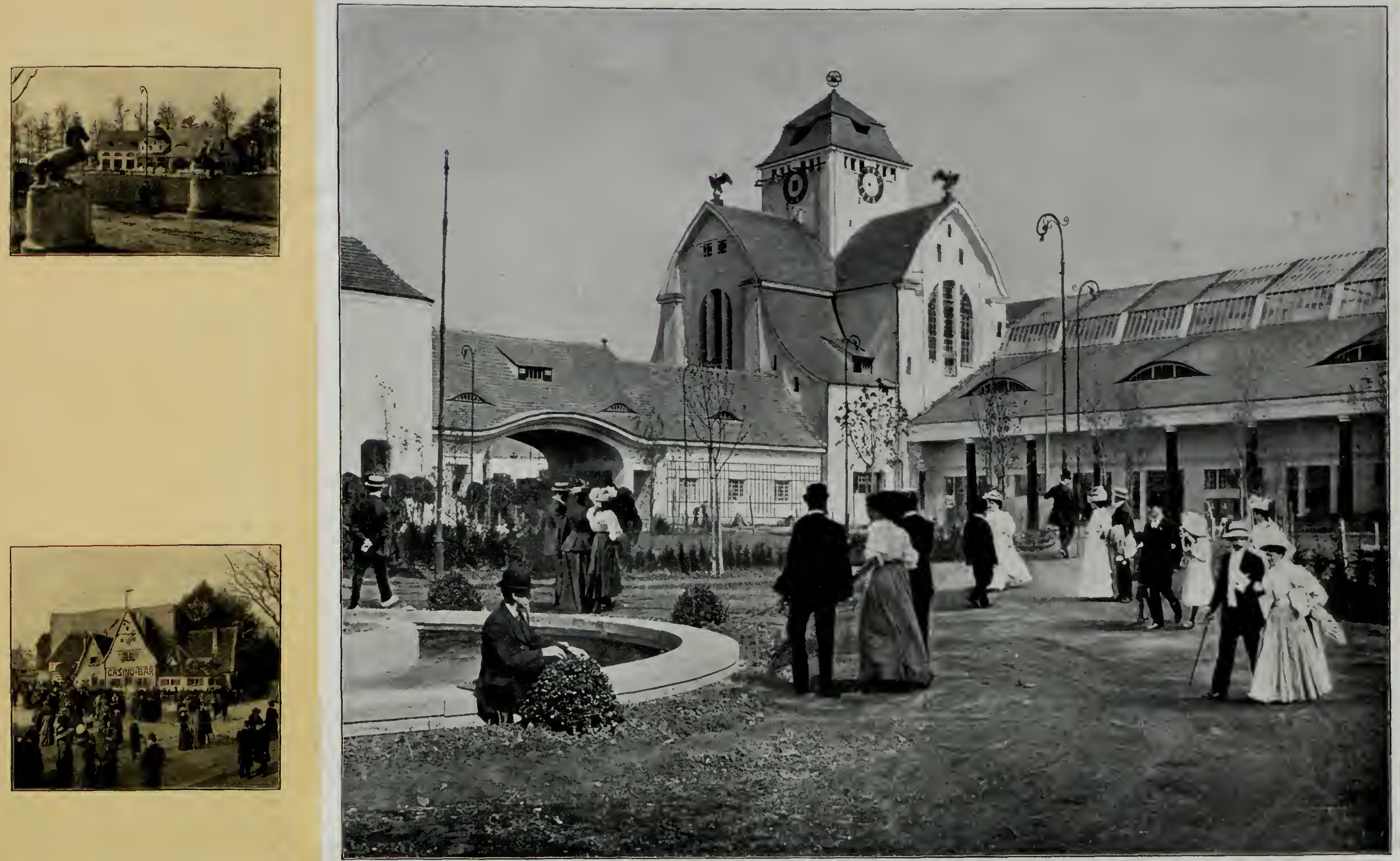

PAVILLON ALLEMAND

Ceci peut être reconnu immédiatement comme une partie de la Section allemande par suite de la caractéristique nationale introduite dans l'architecture. La petite vue du haut représente un coin du Jardin allemand avec la brasserie "Münchener Haus » dans le fond et la petite vue dans le bas le restaurant "Vieux Düsseldorf ». 


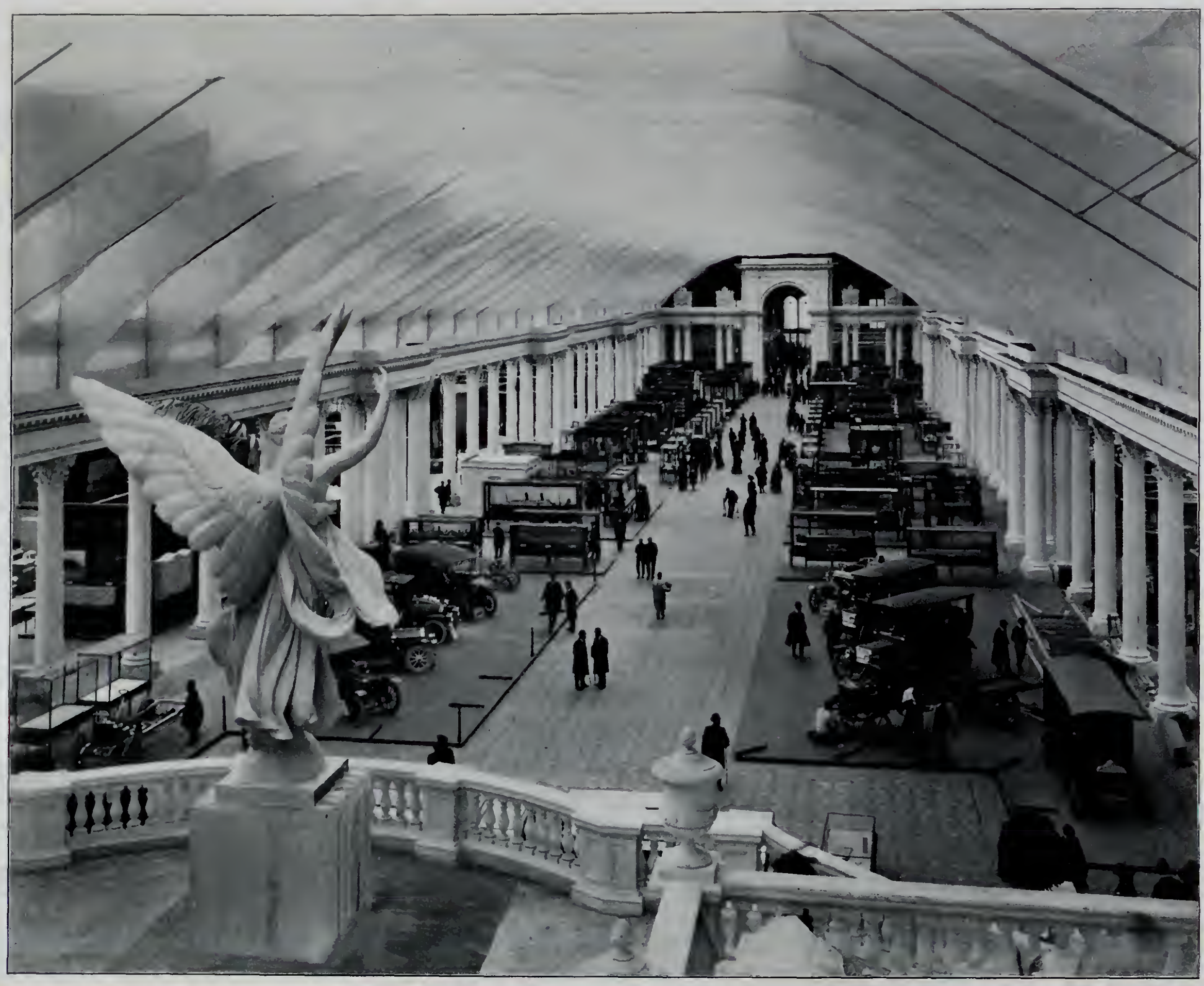

SECTION BRITANNIQUE

Cette vue est prise du pont qui sépare les Sections britannique et française. Elle représente la galerie centrale, mais plusieurs autres galeries se trouvent de chaque côté. 


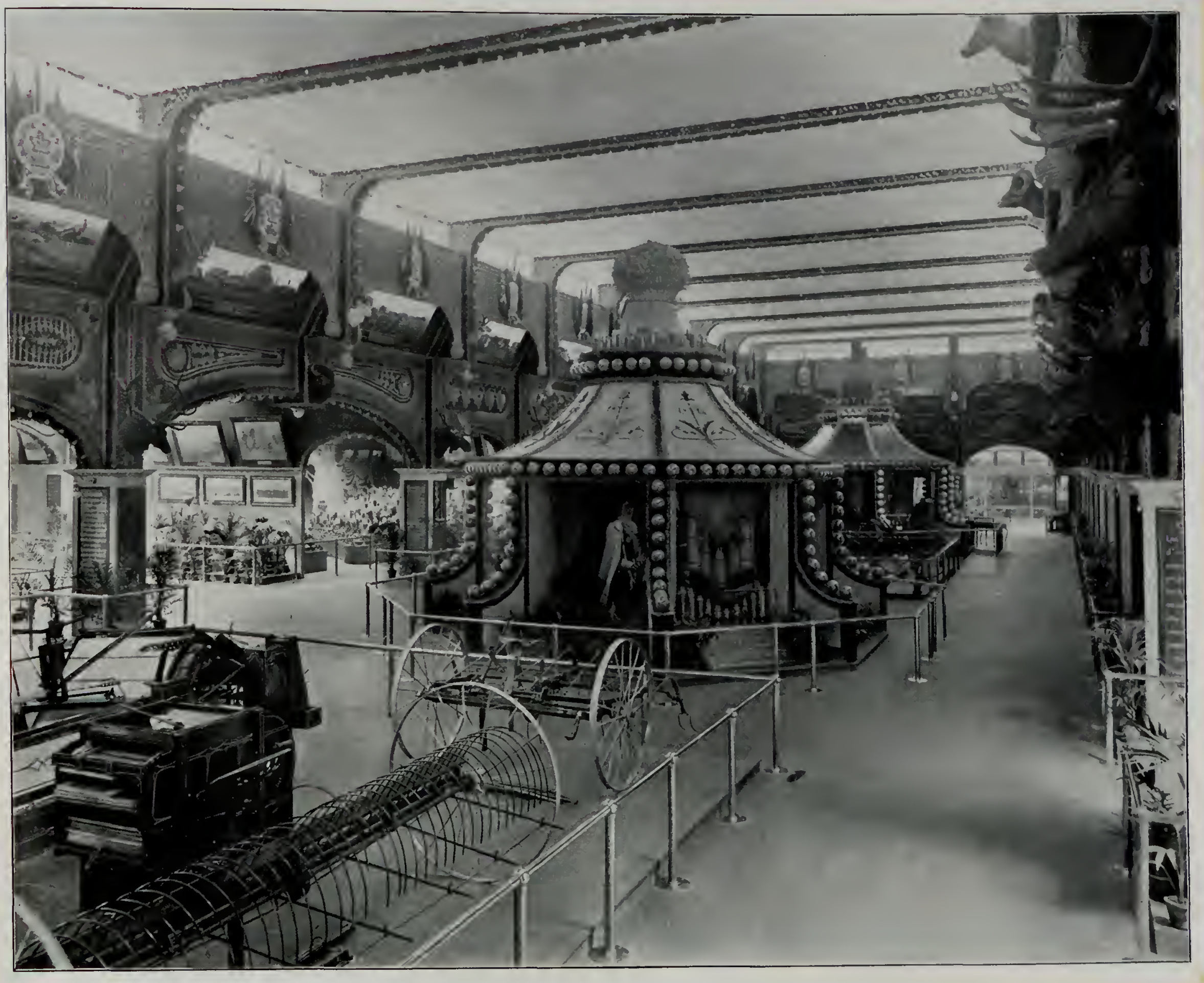

INTÉRIEUR DU PAVILLON CANADIEN

Cette galerie se distingue par le caractère pratique de sa décoration. On y voit des produits des manufactures de ce pays ainsi que les remarquables produits naturels du sol. 


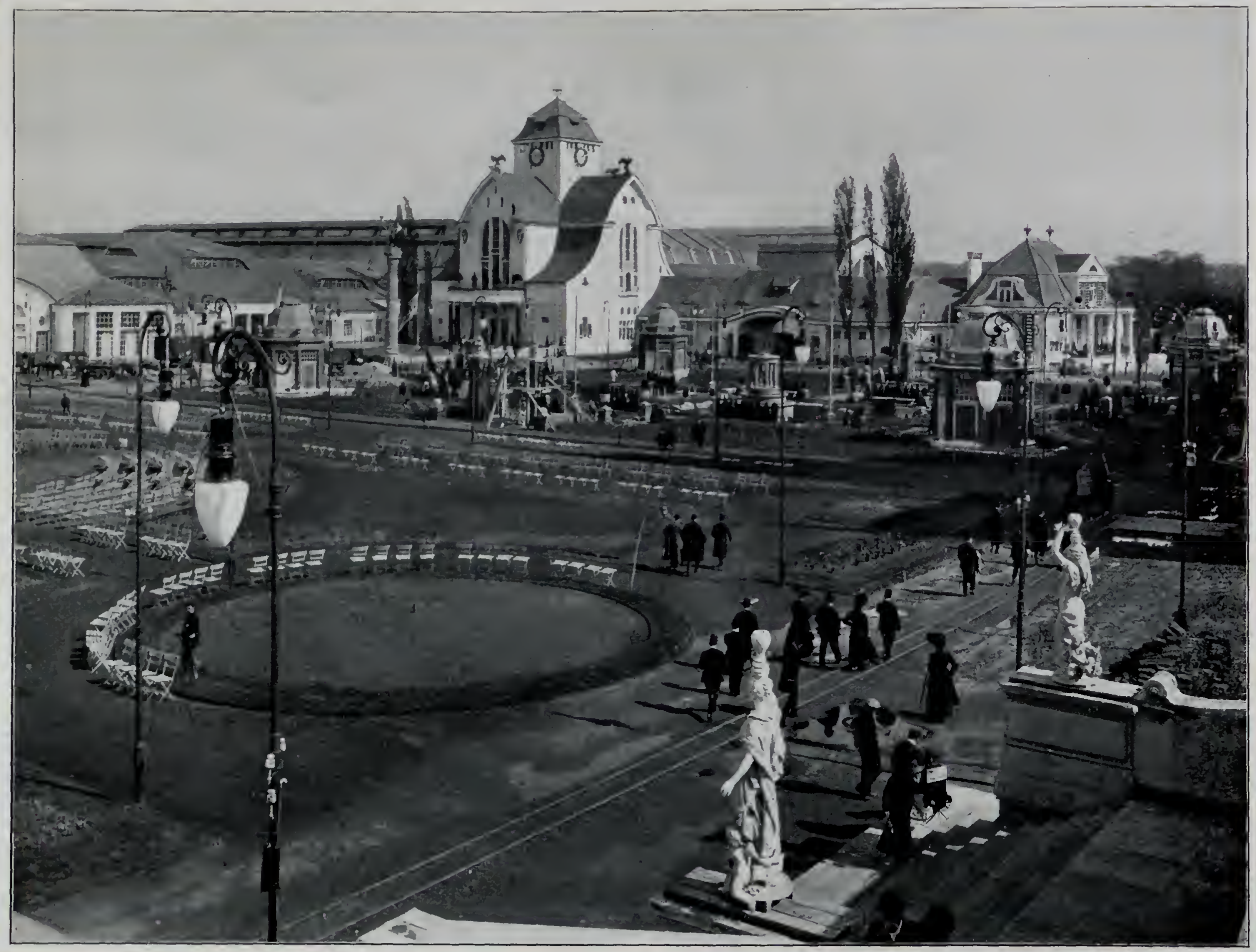

VUE GÉNÉRALE DE LA SECTION ALLEMANDE

Une grande variété d'arbres se trouve dans ces jardins, dont la plupart sont de provenance hollandaise. A droite se trouve le restaurant allemand « Kaiserhof ». 

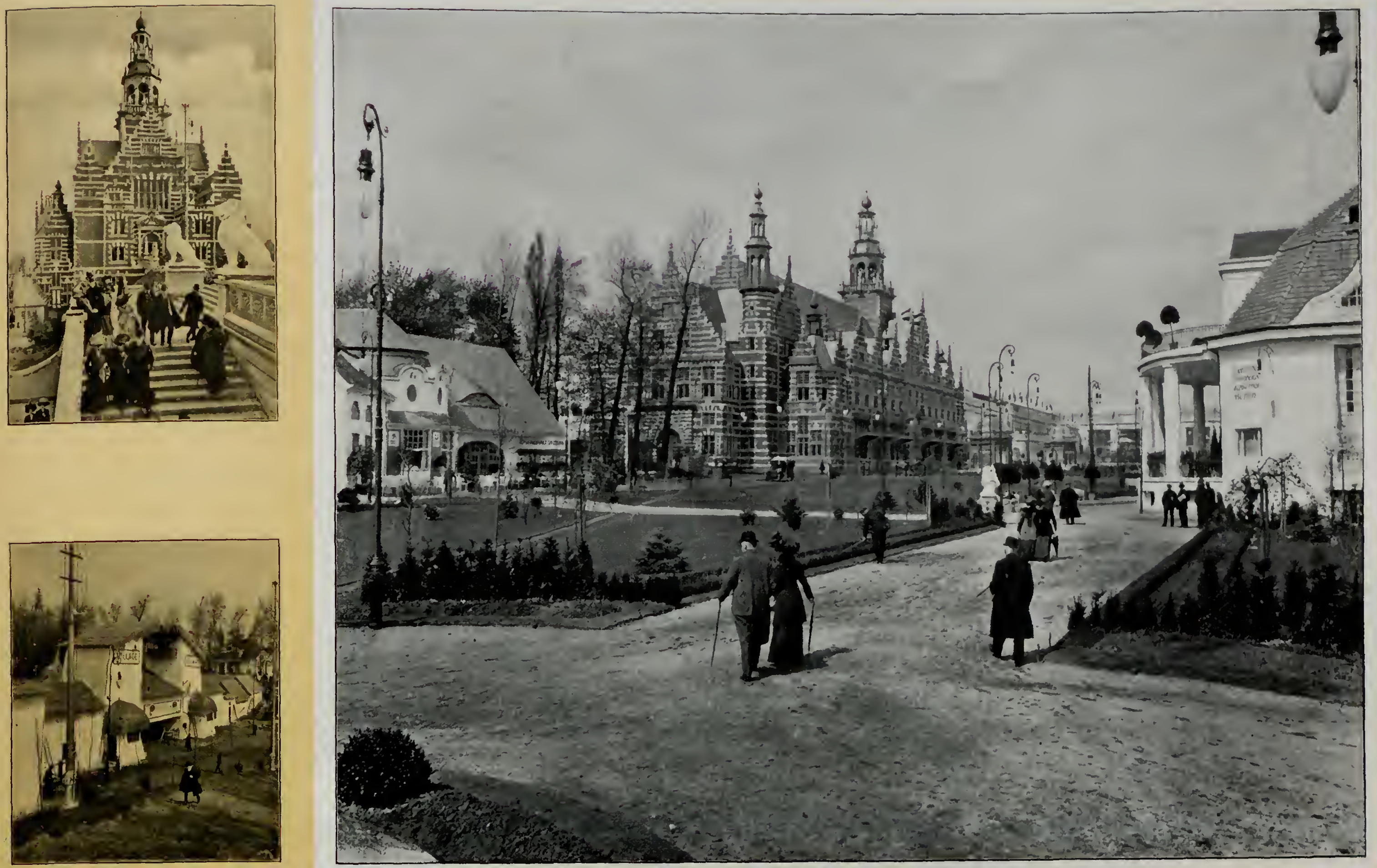

VUE GÉNÉRALE DU PAVILLON HOLLANDAIS

L'architecture est extraordinairement typique et nationale. La petite vue au-dessus montre l'autre côté du pavillon, et la petite vue en-dessous représente le Village Sénégalais qui se trouve à proximité de la Section néerlandaise. 


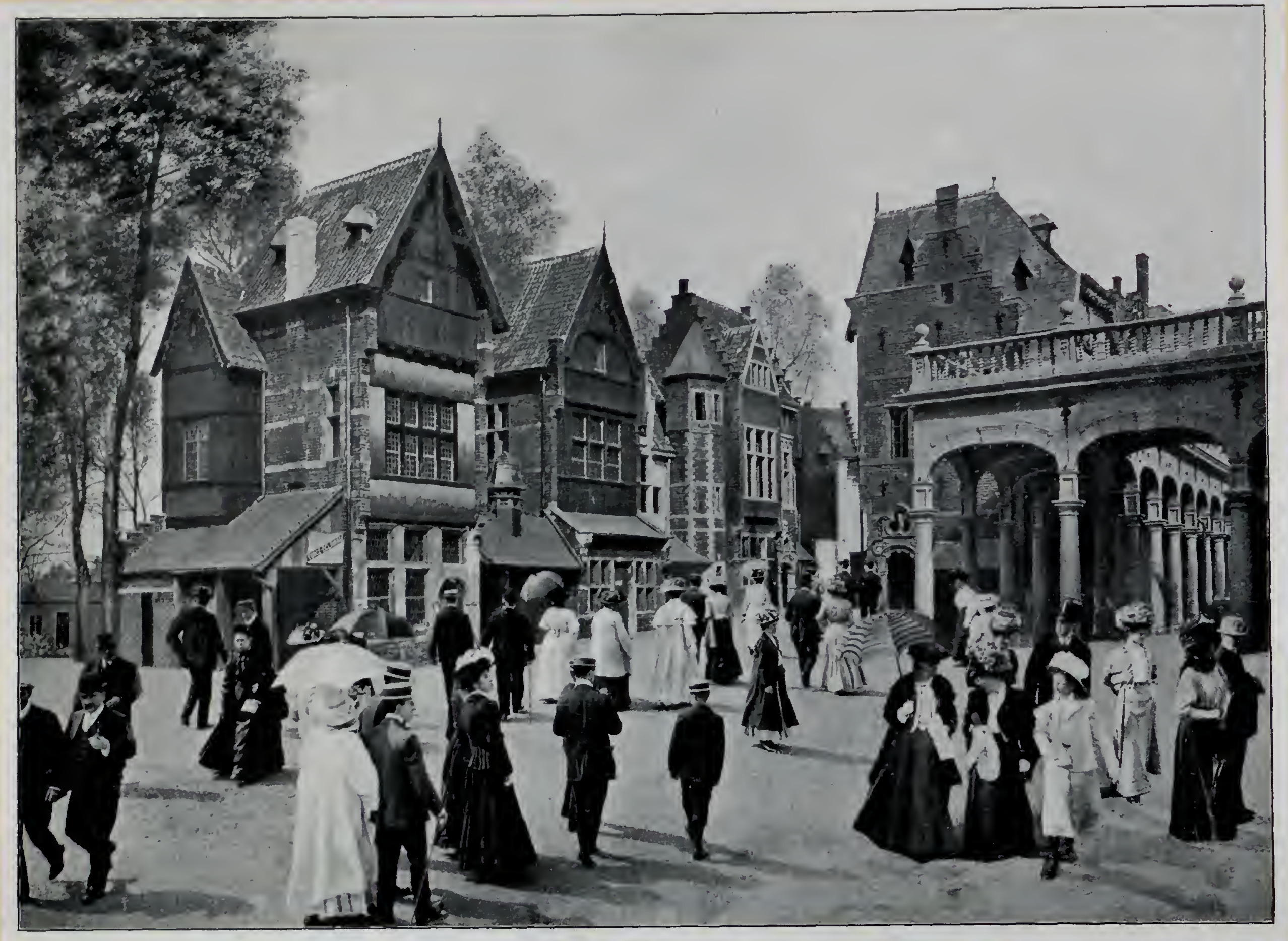

BRUXELLES KERMESSE

Vue prise de l'entrée principale qui est la reconstitution du Vieux Bruxelles. A droite on remarque la galerie entourant le Vieux Marché. 



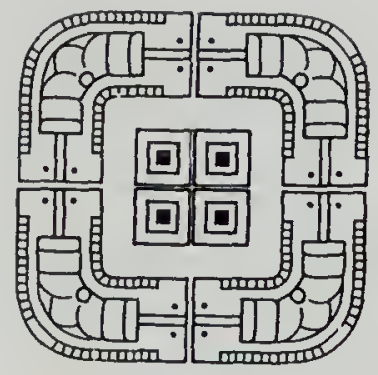




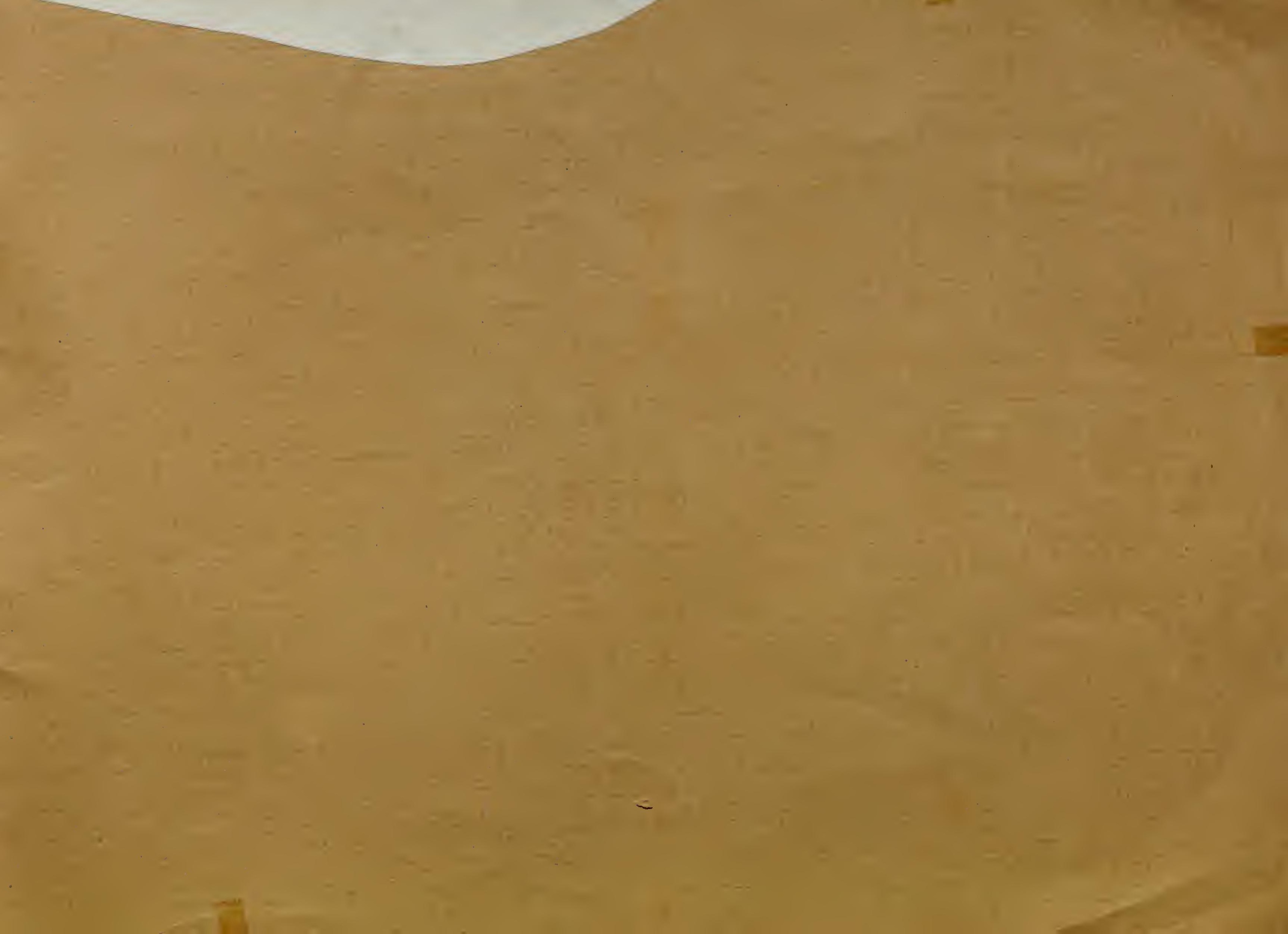


